\title{
A theoretical analysis of the surface dependent binding, peeling and folding of graphene on single crystal copper
}

\author{
Xinghua Shi *, Qifang Yin, Yujie Wei * \\ State Key Laboratory of Nonlinear Mechanics, Institute of Mechanics, Chinese Academy of Sciences, Beijing 100190, People's Republic of China
}

\section{A R T I C L E I N F O}

Article history:

Received 25 November 2011

Accepted 28 February 2012

Available online 8 March 2012

\begin{abstract}
A B S T R A C T
The binding, peeling and folding behavior of graphene on different surfaces of single crystal copper were examined theoretically. We show that the binding energy is the highest on the $\mathrm{Cu}(111)$, and follows the order of $\mathrm{Cu}(111)>(100)>(110)>(112)$. Conventional theory is capable of capturing the dynamic process of graphene peeling seen from molecular dynamics simulations. We show that the number of graphene layers on $\mathrm{Cu}$ surfaces could be distinguished by performing simple peeling tests. Further investigation of the folding/ unfolding of graphene on $\mathrm{Cu}$ surfaces shows that $\mathrm{Cu}(111)$ favors the growth of monolayer graphene. These observations on the interaction between graphene with single crystal $\mathrm{Cu}$ surfaces might provide guidelines for improving graphene fabrication.
\end{abstract}

(c) 2012 Elsevier Ltd. All rights reserved.

\section{Introduction}

Being the unique two-dimensional atomistic crystal, graphene has attracted significant interest in both scientific and technologic communities because of its remarkable electronic [1,2], thermal [3] and mechanical properties [4,5]. High electron mobility, tunable band gaps [6,7], high thermal conductivity, as well as high in-plane stiffness make graphene a promising material for innovative electronic applications. For its industrial level application, mass production of high quality graphene becomes the key. It has been foreseen that epitaxial growth of graphene on various substrates could be a promising route to achieve the goal [8-11]. So far, monolayer and multilayer graphenes are synthesized on copper or nickel surfaces through chemical vapor deposition (CVD) [9-11], and the area of CVD synthesized graphene could reach $1 \mathrm{~cm}^{2}$. Current success in growing graphene on metal surfaces may pave a new road towards its practical applications $[9,11]$. However, it is also reported that with this method, grain boundary defects form within graphene [12,13], which greatly affects the physical properties of graphene [14-16]. The formation of grain boundaries is complicated and we believe the interaction of small graphene islands with metal substrate has considerable contribution, which is not addressed in detail so far. In addition to monolayer graphene, multilayer graphene is found to grow on metal surfaces [17], making it difficult to be distinguished during transfer. The folding of graphene edge is also commonly found experimentally [18-21] which possesses unusual electronic properties $[18,22]$ and mechanical stability [23]. It is shown that in solvent, the folding of suspended graphene has preferred folding angle which directly influences its stability [19]. On substrate, however, the folding becomes complicated due to the interaction of graphene with substrate. Meanwhile it is believed that such folding of graphene edge is induced by thermal undulation. Yet so far no theoretical model exists to describe it. To understand the mechanism of graphene folding, as well as the formations of multilayer graphene and the grain boundary on substrate, eventually provide guidelines for controllable production, theoretical investigations on interfaces of graphene/graphene and graphene/substrate become necessary.

The transfer of graphene from $\mathrm{Cu} / \mathrm{Ni}$ surfaces to other systems is another challenge. In order to utilize graphene in

\footnotetext{
* Corresponding authors: Fax: +86 1082543977

E-mail addresses: shixh@imech.ac.cn (X. Shi), yujie_wei@lnm.imech.ac.cn (Y. Wei). 0008-6223/\$ - see front matter (c) 2012 Elsevier Ltd. All rights reserved. http://dx.doi.org/10.1016/j.carbon.2012.02.092
} 
nanoelectronics and nanodevices, we often need to combine graphene with other materials, e.g., silicon, which involves transfer of graphene from $\mathrm{Cu} / \mathrm{Ni}$ surfaces. To get free standing graphene, a widely used technique in laboratory is chemical removal of the underlying metal substrate [11,24,25], which is inefficient and uneconomic. Here we explore the possibility of using mechanical peeling to transfer graphene from metal substrates. Compared with the chemical removal approach, the peeling approach is more efficient. Another intriguing phenomenon to show here is the characteristics of folding/ unfolding of graphene layers on different $\mathrm{Cu}$ surface, which might supply clues about how to improve the probability of obtaining monolayer graphene. In what follows, we give a brief description about the simulation methods adopted in this work, and then introduce the simulation results for binding, peeling, and folding sequentially.

\section{Simulation methods}

We carried out a series of molecular dynamics (MD) simulations with graphene on single crystal copper substrate. Reactive empirical bond order potential [26] is used to describe the interaction among carbon atoms, which gives a carboncarbon bond length to be $0.142 \mathrm{~nm}$, and is identical to the experimental result [27]. The embedded atom potential is used to describe $\mathrm{Cu}-\mathrm{Cu}$ interactions [28], which has been proven to be capable of describing the structural and mechanical properties of $\mathrm{Cu}$. We use the classical Lennard-Jones (L-J) potential $\mathrm{V}(r)=4 \varepsilon\left(\sigma^{12} / r^{12}-\sigma^{6} / r^{6}\right)$ to describe interaction between carbon and $\mathrm{Cu}$ atoms, where $r$ is the distance between two atoms, $\varepsilon$ and $\sigma$ are the two L-J parameters. This potential is widely used in the simulations of carbon-Cu complex $[29,30]$ for practical considerations. Here $\varepsilon=0.0168 \mathrm{eV}$ and $\sigma=2.2 \AA$ are selected in all the simulations. We will show shortly that with these parameters, the equilibrium distance between graphene and $\mathrm{Cu}(111)$ surface is $2.24 \AA$, and corresponding interfacial binding energy is $24.8 \mathrm{meV} / \AA^{2}$. Both quantities match well with the results from first-principle calculation [31]. Unless stated otherwise, all simulations are run in an NVT ensemble at temperature $300 \mathrm{~K}$.

\section{Interfacial binding of graphene on single crystal $\mathrm{Cu}$}

A graphene disc with diameter of $20 \mathrm{~nm}$ is initially put onto the $\mathrm{Cu}$ surface in close proximity. Periodical boundary condition (PBC) is applied onto the rectangular Cu crystal in $x$ and $y$ directions (Fig. 1a, in yellow). To avoid boundary effects for graphene, the diameter of graphene is at least $4 \mathrm{~nm}$ smaller than the lateral lengths of $\mathrm{Cu}$ crystal. The thickness (along $\mathrm{z}$ direction) of $\mathrm{Cu}$ is greater than $4 \mathrm{~nm}$ and $\mathrm{Cu}$ atoms at the bottom layer are fixed in all the simulations. To investigate the interfacial properties of graphene on different $\mathrm{Cu}$ surface, we simulate the cases of graphene on $\mathrm{Cu}(111),(100),(110)$, and (112) surfaces, respectively. In each set of simulations, graphene is arranged and constrained at a specific angle $\theta$, which is the intersection angle between the armchair edge of the graphene and the horizontal direction of the simulation box, as illustrated in Fig. 1b. To obtain the binding energy between graphene and $\mathrm{Cu}$ surfaces, we first relax the sample at the temperature of $300 \mathrm{~K}$ for $10 \mathrm{ps}$, and then perform an energy minimization at $0 \mathrm{~K}$. By adjusting $\theta$, we establish the dependence of binding energies on $\theta$, from which we find the orientation state bearing the strongest binding energy.

Fig. 1a shows the final structure of graphene adhered onto $\mathrm{Cu}(111)$ with the orientation angle of $\theta=0^{\circ}$. The Moiré pattern, which is a result of overlaying the hexagonal lattices of graphene with hexagonal lattices of $\mathrm{Cu}(111)$, is observed. The periodicity of the pattern is around $6.62 \mathrm{~nm}$, consistent with the experimental observation [32]. Fig. 2a shows the binding energy of the simulated systems as a function of $\theta$ for graphene adhering on $\mathrm{Cu}(111)$. To eliminate possible edge effects, carbon atoms within the edge ring (Fig. 1a, in red) are not accounted in the calculations. The strongest binding energy locates at $\theta=0^{\circ}$, indicating that $0^{\circ}$ orientation of the graphene on $\mathrm{Cu}(111)$ is the most energetically favorable one. Remaining results for the same graphene on $\mathrm{Cu}(100)$, (110) and (112) are shown from Fig. 2b-d, respectively. On $\mathrm{Cu}(110)$ and (112), the graphene also has specific orientation angles with relatively deep well. On the other hand, the energy profile for graphene on $\mathrm{Cu}(100)$ varies mildly with the orientation angle, suggesting graphene on this surface may have no preferred orientation. In other words, the energy required to rotate graphene on $\mathrm{Cu}(100)$ is relatively low. In CVD, graphene islands with different orientations may nucleate and grow. To manufacture graphene with minimum fraction of grain boundaries by using CVD, it is crucial to ensure those graphene islands with few or no misorientation. From this aspect, it is desired that there is a specific direction match between graphene and $\mathrm{Cu}$ where its binding energy is significantly lower than other combinations.

We further find the obtained strength of binding energy is in the order of $(111)>(100)>(110)>(112)$ (Table 1). It is seen that on (111) the binding energy is the largest, about $50 \%$ larger than that on (112). We emphasize here that the binding energy on $\mathrm{Cu}(111)$ agrees well with the first-principle calculation [31], indicating the parameters selected for the L-J potential are reasonable. Existing experiments showed that largearea monolayer graphene could grow on $\mathrm{Cu}(111)$ and $\mathrm{Cu}(100)[10,11]$, which suggests that graphene prefers to grow on surfaces owing high binding energy. We will show shortly that the high binding energy between graphene and $\mathrm{Cu}$ surface is necessary for the growth of monolayer graphene.

\section{Peeling of graphene from $\mathrm{Cu}$ surfaces}

Another feasible way to capture the binding property of graphene on substrate is to perform peeling tests, which is a commonly used method to measure the interfacial properties of thin films [33,34]. As shown in Fig. 3a (the inset), with an applied peeling force, one edge of graphene is peeled off the substrate at a peeling angle $\beta$. Conventionally the peeling force and peeling angle are connected by peeling equation [35] $F(1-\cos \beta)+F^{2} / 2 E t-\gamma=0$, where $F$ is the peeling force per unit length, $E$ is the Young's rigidity of graphene, $t$ is the thickness of graphene and $\gamma$ the binding energy per unit area. The elastic energy, the second term in the equation, is usually negligible compared with the other two terms. The equation is thus reduced to be $F(1-\cos \beta)-\gamma=0$. 
(a)

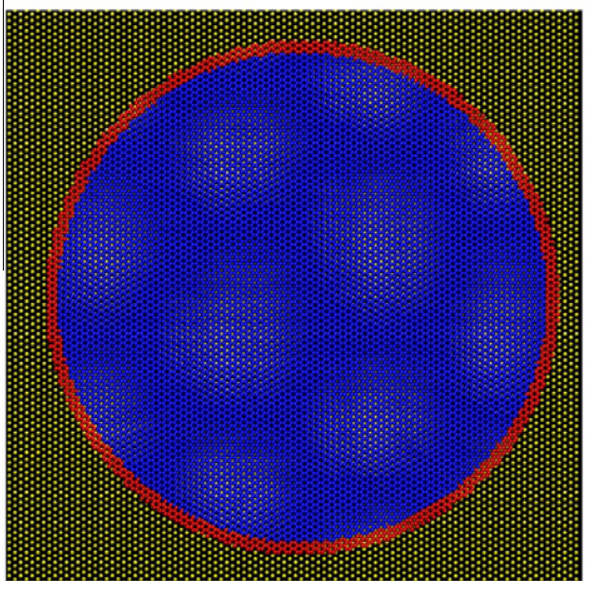

(b)

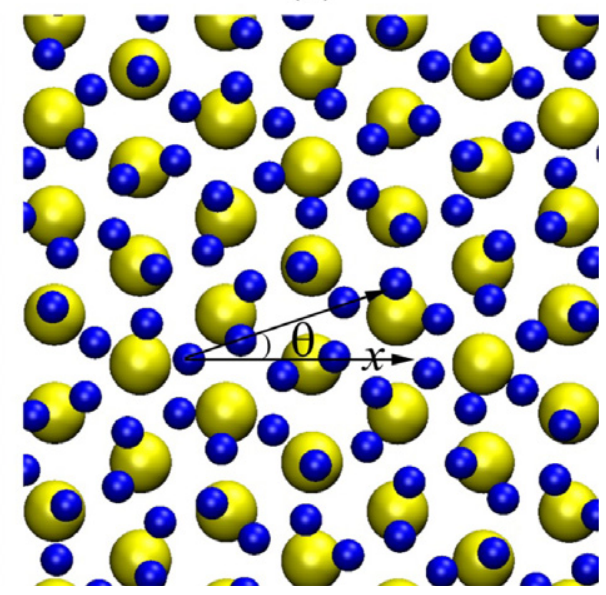

Fig. 1 - (a) A graphene disc (in blue) on $\mathrm{Cu}(111)$ surface (in yellow). (b) Close view of graphene on $\mathrm{Cu}(111)$. Angle $\theta$ is defined as the angle between the armchair edge and the horizontal direction. (For interpretation of the references to color in this figure legend, the reader is referred to the web version of this article.)

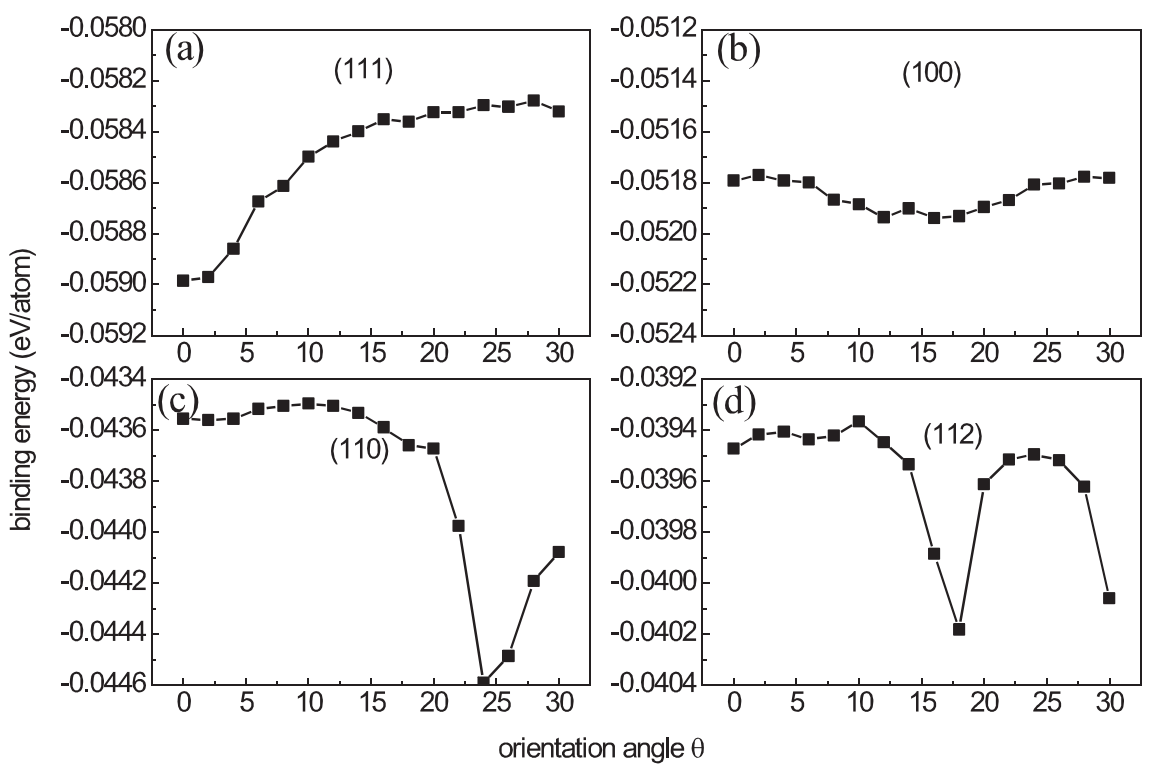

Fig. 2 - The binding energy normalized by the number of carbon atoms as a function of orientation angle of a same graphene disc on (a) $\mathrm{Cu}(111),(b) \mathrm{Cu}(100)$, (c) $\mathrm{Cu}(110)$ and (d) $\mathrm{Cu}(112)$.

Table 1 - The binding energy of graphene on various Cu surfaces at specific orientation angle and peel-off force of graphene at peeling angle of $\beta=90^{\circ}$, orientation angle $0^{\circ}$.

\begin{tabular}{lccc} 
Cu surface & $E_{\text {bind }}\left(\mathrm{meV} / \AA^{2}\right)$ & Orientation angle $\left(^{\circ}\right)$ & $f_{\text {peel }}\left(\mathrm{meV} / \AA^{2}\right)$ \\
\hline$(111)$ & 24.8 & 0 & 23.67 \\
$(100)$ & 21.91 & 16 & 21.92 \\
$(110)$ & 18.43 & 24 & 19 \\
$(112)$ & 16.73 & 18 & 16.2 \\
\hline
\end{tabular}

To check the validity of the conventional theory for the peeling of graphene from Cu substrate, we carried out a series of additional MD simulations. We put one graphene ribbon onto the (100) surface of a rectangular Cu crystal with the orientation angle $0^{\circ}$. Typical simulation boxes for the graphene-
$\mathrm{Cu}$ system have a dimension of $1.75 \mathrm{~nm} \times 91.1 \mathrm{~nm} \times 10 \mathrm{~nm}$. $\mathrm{PBC}$ is applied onto the planar directions of the interface. Carbon atoms at one edge of the graphene are clamped by constraining their relative positions. The clamped part is then connected with a spring with a spring constant $k=10 \mathrm{eV} / \AA^{2}$, 

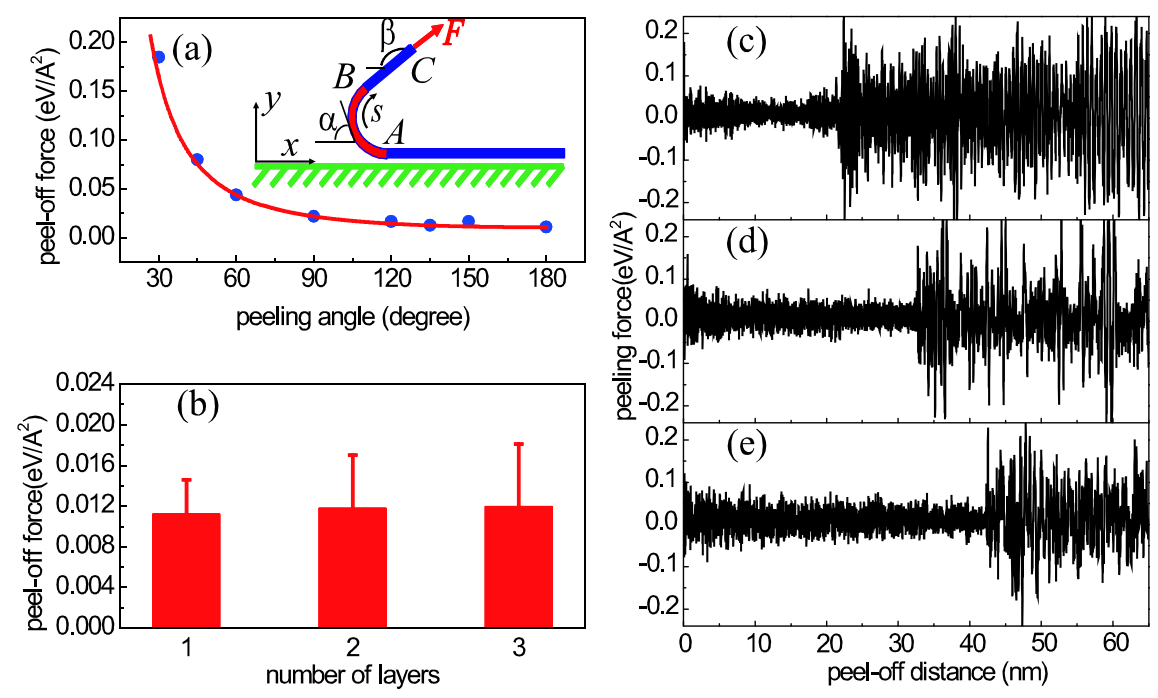

Fig. 3 - Characteristics of the peeling force as the graphene is peeled off from the $\mathrm{Cu}(100)$ surface with the orientation angle $0^{\circ}$. (a) The peel-off force as a function of the peeling angle. The blue dots are the results from our MD simulations and red line from the theory [35] where $\gamma=21.91 \mathrm{meV} / \AA^{2}$. A Fast-Fourier-Transformation (FFT) filter with the window width $5 \mathrm{ps}$ is performed to the raw data to obtain the averaged peel-off force. The inset is a schematic to show that the graphene is peeled off at the peeling angle $\beta$ under the loading force $F$. (b) The peel-off force as a function of the number of the layers in graphenes. (c-e) The peeling force as a function of the peel-off distance for (c) a monolayer graphene, (d) a bilayer graphene, and (e) a trilayer graphenes at temperature $T=10 \mathrm{~K}$. (For interpretation of the references to color in this figure legend, the reader is referred to the web version of this article.)

and we pull the other end of the spring at a velocity $\mathrm{v}=0.01 \mathrm{~nm} / \mathrm{ps}$. Another edge of the graphene is set free during the simulations.

Numerical tests for a series of peeling angles are conducted with the results presented in Fig. 3a (blue dots). It is seen that the simulated results agree well with the theoretical prediction (Fig. 3a, red line) with a binding energy of $\gamma=21.91 \mathrm{meV} / \AA^{2}$. From the results we get the information that the conventional peeling theory still works well for nanoscale graphene. So experimentally the peeling test can be used to get the interfacial properties of graphene on $\mathrm{Cu}$ surfaces (see Table 1). Furthermore, such approach can be extended to investigate the interfacial properties of graphene on other metal surfaces.

So far we have not studied the role of the mechanical property of graphene itself during peeling. By neglecting the elastic term, the theoretical prediction still agrees well with the results from the MD simulations in peeling tests, indicating that the in-plane tension of graphene has negligible effect on the peeling force (Fig. 3a). A natural question is whether the bending stiffness of graphene plays some role during peeling tests. From the peeling equation we can see that there is no such term associated with the bending of graphene. It seems that the bending stiffness has no influence on the peeling force of graphene. To demonstrate it, we carry out additional simulations with the monolayer graphene replaced by bilayer and trilayer graphenes, which effectively adjusts the bending rigidities of graphene. Fig. $3 \mathrm{~b}$ shows the peeling forces of monolayer, bilayer and trilayer graphenes from the $\mathrm{Cu}(100)$ at peeling angle of $\beta=180^{\circ}$. It is seen that the peeling forces for graphene with different layers are almost identical. The results indicate that based on the experimentally measured peeling force, we cannot make any judgement on the number of layers of graphene peeled-off. Thus it becomes impractical to differentiate the number of graphene layers peeled-off by peeling forces from peeling tests. We suggest next a simple approach to distinguish the layers of graphene on the substrate.

As the graphene(s) being peeled off the substrate at one special peeling angle of $\beta=180^{\circ}$ and at temperature $\mathrm{T}=10 \mathrm{~K}$, we find the intervals between the graphene peeled-off and the one on substrate increase with the number of layers of graphene. It is also seen from Figs. 3c-e that the peeling forces become highly oscillated at some specific peel-off distances which is defined as the distance between the initial and the final positions of point $C$ in Fig. 3a. Interestingly we find the positions where the oscillation of peeling force starts increase with the number of layers in graphene (Fig. 3c-e): they are about 22, 33 and $42 \mathrm{~nm}$ for the monolayer, bilayer and trilayer graphenes, respectively. This phenomenon is attributed to sticking and slipping between the peeled graphenes and that still residing on substrate. As shown in Fig. 4a, when the graphene is peeled off from state $i$ to state ii at the peeling angle of $\beta=180^{\circ}$, it is parallel to the substrate due to the peeling force. The interval of $d$ between the two portions of the graphene can be calculated as following. Suppose the graphene is peeled off at peeling angle of $\beta$ due to peeling force of $F$ (Fig. 3a, inset), the deformation curve of graphene peeled-off consists of two regimes: segment $A B$, which is considered to be under pure bending and clamped at point $\mathrm{A}$, and segment $B C$, which is considered to be straight and under pure stretching. The curve $\mathrm{AB}$ can be described by $\mathrm{Dd}^{2} \alpha / \mathrm{ds}^{2}-\mathrm{F}$ $\sin \beta-\alpha=0$ where $D$ is the bending stiffness of graphene, $s$ is the arc length along the deformed graphene and $\alpha$ is the 
(a)

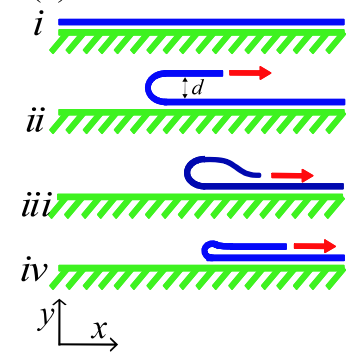

(b)

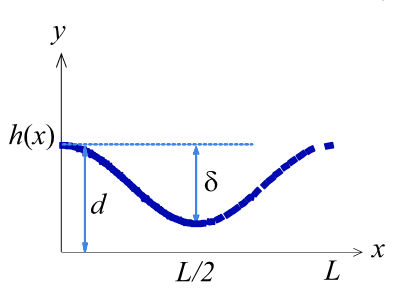

(c)

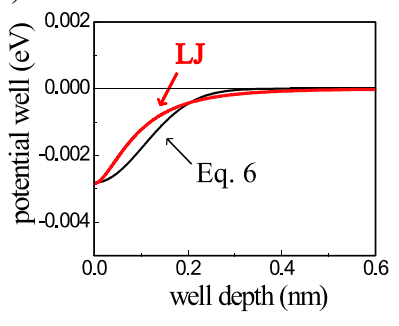

Fig. 4 - (a) Schematic show the process of graphene on Cu surface (ii) being peeled at $\beta=180^{\circ}$, (iii) sticks onto the surface and (iv) slips on the surface. (b) Schematic show the undulated graphene extended in $x$-direction. The transverse deflection $h(x)$ is assumed to depend only on $x$. The slope of the end of graphene at $x=0, L / 2$ are constrained to be zero. (c) Schematic show of Lennard-Jones potential and that described in Eq. (6). In L-J the parameters are selected as $\sigma=0.34 \mathrm{~nm}$ and $\varepsilon=0.00284 \mathrm{eV}$. The material parameters in Eq. (6) are set to $E_{a}=0.11 k_{\mathrm{B}} \mathrm{T}$ and $\Omega=93.75 \mathrm{k}_{\mathrm{B}} \mathrm{T} / \mathrm{nm}^{2}$. For comparison the position of well of L-J is shifted to zero. (For interpretation of the references to color in this figure legend, the reader is referred to the web version of this article.)

angle between the tangent of graphene at $\mathrm{s}$. The boundary conditions at point $\mathrm{A}$ and $\mathrm{B}$ are $\alpha_{\mathrm{A}}=\pi, \mathrm{y}_{\mathrm{A}}=0, \alpha_{\mathrm{B}}=\pi-\beta$, and $\left.\frac{d \alpha}{d s}\right|_{B}=0$. Following the approach in [36], we obtain $\frac{d \alpha}{d s}=$ $\sqrt{\frac{2 F}{D}(1-\cos (\beta-\alpha))}$. This equation can be solved by considering $d x=-\cos \alpha d s, d y=\sin \alpha d s$, as well as $\beta=\pi$. Segment $A B$ can be described by $x(\alpha)=x_{A}+\sqrt{\frac{D}{2 F}} \int_{\pi}^{\alpha} \frac{\cos \alpha d \alpha}{\sqrt{1+\cos \alpha}}$ and $y(\alpha)=$ $\sqrt{\frac{D}{2 F}} \int_{\pi}^{\alpha} \frac{\sin \alpha d \alpha}{\sqrt{1+\cos \alpha}}$. As $\alpha \rightarrow 0$ at point $B$, we obtain the interval distance of $d=2 \sqrt{D / F}$. Recall when $\beta=\pi$, the peeling equation is simply $F=\gamma / 2$. So the equation of interval distance can be recasted into

$d=\sqrt{\frac{8 D}{\gamma}}$.

Eq. (1) indicates that the interval between the portion of graphene being peeled off and the other part residing on substrate is totally determined by two material parameters: The bending stiffness of the graphene and the binding energy between graphene and its substrate. The interval would increase with the bending stiffness controlled by the number of layers of graphene. The more layers, the larger interval between the two parts of the graphene. So the theoretical result is consistent with the one from MD. Similar expressions have also been obtained to describe the self-folding of free standing carbon nanotubes [37] and graphenes [38].

It should be noted that in the theory, we did not consider the van der Waals (vdW) interaction between the graphene peeled-off and the rest on substrate. Since the bending stiffness of graphene is about $0.11 \mathrm{nN} \cdot \mathrm{nm}$ [39] and the binding energy is about $0.4 \mathrm{nN} / \mathrm{nm}$, the interval $d$ is in the range of $1.5 \mathrm{~nm}$, beyond the range of vdW interaction in MD simulations, which is typically around $1.2 \mathrm{~nm}$. However, with the thermal induced undulation to graphene ribbons, such interval can be overcomed and the graphene once peeled-off might stick to the graphene on the substrate with additional vdW interaction, as illustrated in Fig. 4a, state iii. To understand how thermal undulation influences the sticking position, we adopted an analytical model within the framework of classical statistical mechanics. Consider a peeled-off graphene with length $L / 2$ extending in $x$-direction (Fig. 4b), we could approximate thermal induced deflection in $y$-axis to be

$h(x)=\tau \sum_{i=2,4,6 \ldots}^{n_{\text {even }}} a_{i} \cos \frac{i \pi x}{L}$

where $\tau$ is the width of graphene, $n_{\text {even }}$ is an even number with $n_{m}=n_{\text {even }} / 2$ being the number of modes taken into account, and $a_{i}$ represents a set of $n_{m}$ random variables describing the response of the graphene to thermal excitation, a type of Brownian motion. We note that the deflection satisfies $h^{\prime}(0)=h^{\prime}(L / 2)=h^{\prime}(L)=0$. The bending energy of graphene due to the thermal undulation is then given by

$U_{b}=\frac{1}{2} D \tau \int_{0}^{L / 2} h^{\prime \prime}(x)^{2} d x$.

In the framework of statistical mechanics, when a system reaches a state of thermal equilibrium, the average energy associated with each random variable used to represent one configuration of the system is $\frac{1}{2} k_{B} T$, where $k_{B}$ is the Boltzmann constant and $\mathrm{T}$ the absolute temperature. The mean bending energy of graphene is

$\left\langle U_{b}\right\rangle=\sum_{i=2,4,6 \ldots}^{n_{\text {even }}} D \frac{\pi^{4} i^{4} \tau^{3}}{8 L^{3}}\left\langle a_{i}^{2}\right\rangle=\frac{1}{2} k_{B} T n_{m}$

By using equipartition, we obtain the mean fluctuation amplitude for each mode as

$\sqrt{\left\langle a_{i}^{2}\right\rangle}=\frac{2}{i^{2} \pi^{2}} \sqrt{\frac{l^{3}}{d_{b}}}$,

where $d_{b} \equiv D / k_{B} T$, and $l \equiv L / \tau$. We note that the amplitudes of the modes with large wave numbers are very small, indicating the undulation of graphene is mainly controlled by the modes with small wave numbers. With possible approaching of the graphenes peeled-off and that residing on substrate by thermal undulation, their by vdW interaction - which was neglected in previous discussion - may contribute energy to the system. For analytical convenience, we adopt the following form to describe the interaction between the two portions of the graphene,

$U_{a}(q)=-k_{B} T \ln \left\lfloor\left(e^{E_{a} / k_{B} T}-1\right) e^{-\left(\Omega / 2 k_{B} T\right) q^{2}}+1\right\rfloor$ 
where $q$ presents the interval between the graphene peeledoff and that on substrate, $E_{a}$ is the depth of the energy well at $q=0$ and $\Omega$ the curvature of the well. This form has been successfully used to represent vdW interactions in other systems, e.g., the interaction between receptor and ligand in a cellular system [40]. To best fit the L-J potential for the graphene-graphene interaction, material parameters in Eq. (6) are set to $E_{a}=0.11 k_{B} T$ and $\Omega=93.75 k_{B} \mathrm{~T} / \mathrm{nm}^{2}$. Fig. $4 \mathrm{c}$ shows the comparison of two potentials.

Suppose the stiction of graphene occurs at the end of graphene, say $\mathrm{x}=\mathrm{L} / 2$, we are interested in the subsequent variants of the random thermal fluctuations at this point. Such thermal fluctuation modes would give rise to an amplitude close to the interval between graphenes, in order to make the stiction possible. Let us consider the first two modes of thermal modes, and the corresponding partition function is

$Z=\int_{-\infty}^{+\infty} \int_{-\infty}^{+\infty} e^{-\left(U_{b}+U_{a}\right) / k_{B} T} d a_{2} d a_{4}$

Recall $q=h(0)-h(L / 2)=\tau\left(a_{2}+a_{4}\right)-\tau\left(a_{4}-a_{2}\right)=2 \tau a_{2}$, we obtain the above integral as

$Z=\frac{l^{3}}{8 d_{b} \pi^{3}}\left[1+\frac{e^{\varepsilon_{a}}-1}{\sqrt{1+\omega l^{3} /\left(\pi^{4} d_{b}\right)}}\right]$

where $\varepsilon_{a} \equiv \frac{E_{a}}{k_{B} T}$ and $\omega \equiv \frac{\Omega \tau^{2}}{k_{B} T}$. The thermal average of the deflection in $y$-axis at point $x=L / 2$, say $\sqrt{\left\langle\delta^{2}\right\rangle}=2 \tau \sqrt{\left\langle a_{2}^{2}\right\rangle}$, is given by

$\left\langle\delta^{2}\right\rangle=\frac{1}{Z} \int_{-\infty}^{+\infty} \int_{-\infty}^{+\infty} q^{2} e^{-\left(\mathrm{U}_{b}+\mathrm{U}_{a}\right) / k_{B} \mathrm{~T}} d a_{2} \mathrm{da} a_{4}$.

which could be explicitly expressed as

$\frac{\delta^{2}}{d^{2}}=\frac{\tau^{2} l^{3}}{d^{2} \pi^{4} d_{b}} \frac{e^{\varepsilon_{a}}-1+\left(1+\omega l^{3} /\left(\pi^{4} d_{b}\right)\right)^{3 / 2}}{\left(1+\omega l^{3} /\left(\pi^{4} d_{b}\right)\right)\left[e^{\varepsilon_{a}}-1+\left(1+\omega l^{3} /\left(\pi^{4} d_{b}\right)\right)^{1 / 2}\right]}$

Eq. (10) measures the ratio between the standard deviation of wave amplitudes in $y$-axis by thermal fluctuation and the interval between the two parts of the graphene (Eq. (1)). To ensure successful stiction, $(\delta-t) / d$ has to be larger than one, where $t=0.34 \mathrm{~nm}$ is the interfacial distance between two sticked graphenes. The contour plots in Figs. 5 show the dependence of $\delta / d$ on $l$ (the length of graphene peeled-off) and $D$ (the bending stiffness) for $\varepsilon_{a}=0.11, \omega=2.93$, $\gamma=0.4 \mathrm{nN} / \mathrm{nm}$ and $\tau=0.125 \mathrm{~nm}$. With constant bending stiffness, it is seen that thermal undulation is more effective to ensure stiction if graphene peeled-off is longer, see Fig. 5a and $5 \mathrm{~b}$. Likewise, for a fixed peeled-off length, thermal undulations is easier to induce stiction in graphene with lower bending stiffness. The level curve corresponding to $(\delta-t) /$ $d=1$ represents the marginal situation: The stiction would not occur if the material parameters locate higher than the curve, on the contrary, the stiction would occur if they locate lower than the curve. We emphasize that the theoretical predictions agree well with the MD simulation results if the bending stiffness of monolayer, bilayer and trilayer graphenes is selected as $D=0.11,0.22,0.33 \mathrm{nN} \cdot \mathrm{nm}$. We note that the relationship between bending stiffness and number of layers does not follow the one described by Zhang et al. [41]. In
[41], due to the clamped constraints at both edges, all the monolayers are confined within the multilayered graphene system. Such confinement greatly enhances the bending stiffness. In current work, those monolayers can slide with respect to each other. The bending stiffness is thus approximately proportional to the number of layers in multilayer graphene. The temperature also influences the stiction. At $\mathrm{T}=300 \mathrm{~K}$, the bending stiffness of graphene sticked due to thermal undulation is in the range of $1-5 \mathrm{nN} \cdot \mathrm{nm}$ (Fig. 5b), which is roughly one order of magnitude larger than that at $\mathrm{T}=10 \mathrm{~K}$ (Fig. 5a). Fig. $5 \mathrm{c}$ shows the variation of stick position with temperatures. It is seen that the position decreases as temperature increases, indicating the stiction would happen more quickly with the help of higher level thermal undulation.

\section{Folding/unfolding of graphene on $\mathrm{Cu}$ surfaces}

Once the edge of graphene comes into contact with the substrate, the stiction propagates leftwards quickly, eventually almost the whole part of graphene peeled-off sticks onto the graphene on substrate (Fig. 4a, state iv). Since then on, the lateral force here consists of two parts: one is the force needed to balance the net binding forces of graphene/graphene and graphene/substrate; the other is to balance the friction of graphene/graphene. The friction is attributed to the interlayer interaction as well as the edge barrier [42]. It is highly oscillating, which is the characteristics of atomistic friction and has been extensively studied elsewhere [43-45]. The study on the net binding forces of graphene/graphene and graphene/substrate has not been addressed. Without applied loading, the binding force of graphene/graphene tends to fold the graphene on the substrate, while the binding force of graphene/substrate tends to resist folding. If the binding force of graphene/ graphene is large enough to overcome the net force of binding force between graphene/substrate and friction between graphene/graphene, the stiction of graphenes will spontaneously drive the formation of bilayer graphene on the substrate. In contrast, if the binding force of graphene/substrate is large enough, such folding would not occur. We have done a series of MD simulations with pre-folded graphene on different $\mathrm{Cu}$ surfaces. At $\mathrm{T}=300 \mathrm{~K}$, the results show that on $\mathrm{Cu}(111)$, the folded graphenes spontaneously unfold to become monolayer graphene (Fig. 6a and b), while on $\mathrm{Cu}(112)$, the folded part of graphene continues propagating ahead, eventually form bilayer graphene on the surface (Fig. 6c and d). In Section 3, we show that $\mathrm{Cu}(111)$ has much higher binding energy than $\mathrm{Cu}(112)$, indicating that the surface with high binding energy can grow unfolded graphene. On $\mathrm{Cu}(100)$ and (110) surfaces, neither folding nor unfolding happens, indicating the net force cannot overcome the friction force. These results again suggest that $\mathrm{Cu}(111)$ is preferred to be used in fabricating monolayer graphene.

\section{Conclusions}

We investigated the binding energy of graphene on different surfaces of single crystal $\mathrm{Cu}$. We found that binding energies 
(a)

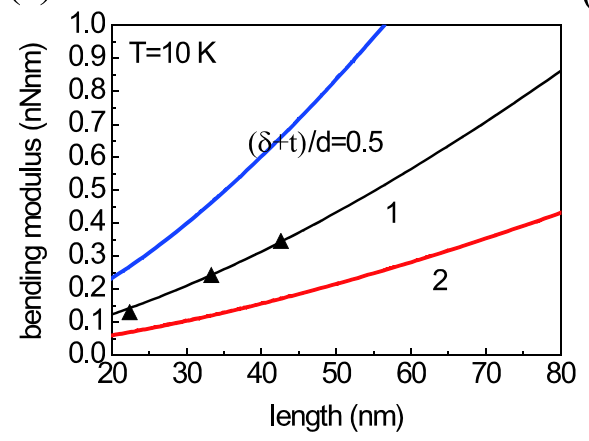

(b)

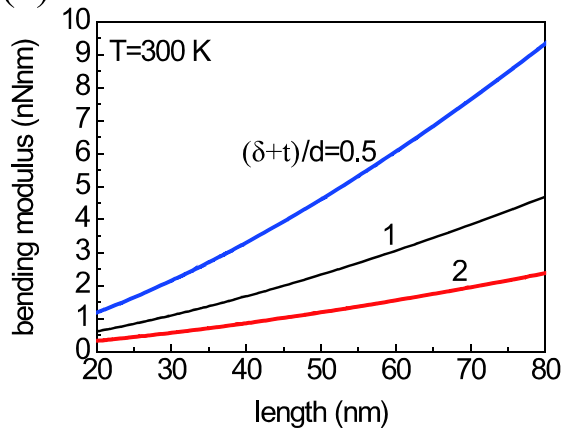

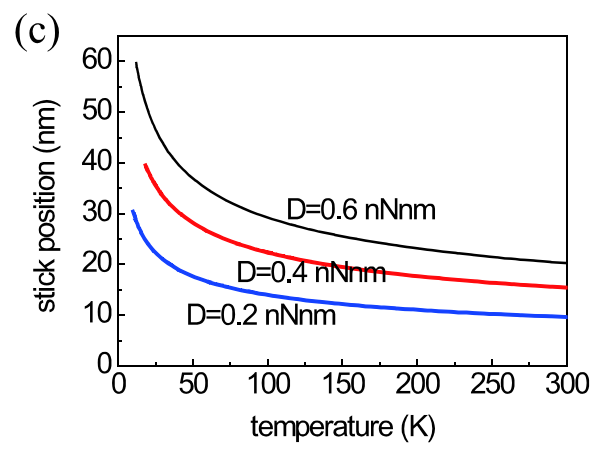

Fig. 5 - (a,b) Level curves of the ratio of standard deviation $\delta$ of graphene fluctuation at the sticking point normalized by the interval $d$ of graphenes over the plane of length of graphene peeled-off, $L$, and bending stiffness of $D$. The parameters are selected as $\varepsilon_{a}=0.11, \omega=2.93, \gamma=0.4 \mathrm{nN} / \mathrm{nm}, \tau=0.125 \mathrm{~nm}$. The curves correspond to the values $(\delta+\mathrm{t}) / d=0.5$ (blue), 1.0 (black), 2.0 (red). The triangles are results from MD simulation. (c) Variation of stick position with the temperature. The curves correspond to the values $\mathrm{D}=0.6$ (black), 0.4 (red) and $0.2 \mathrm{nN} \cdot \mathrm{nm}$ (blue). (For interpretation of the references to color in this figure legend, the reader is referred to the web version of this article.)
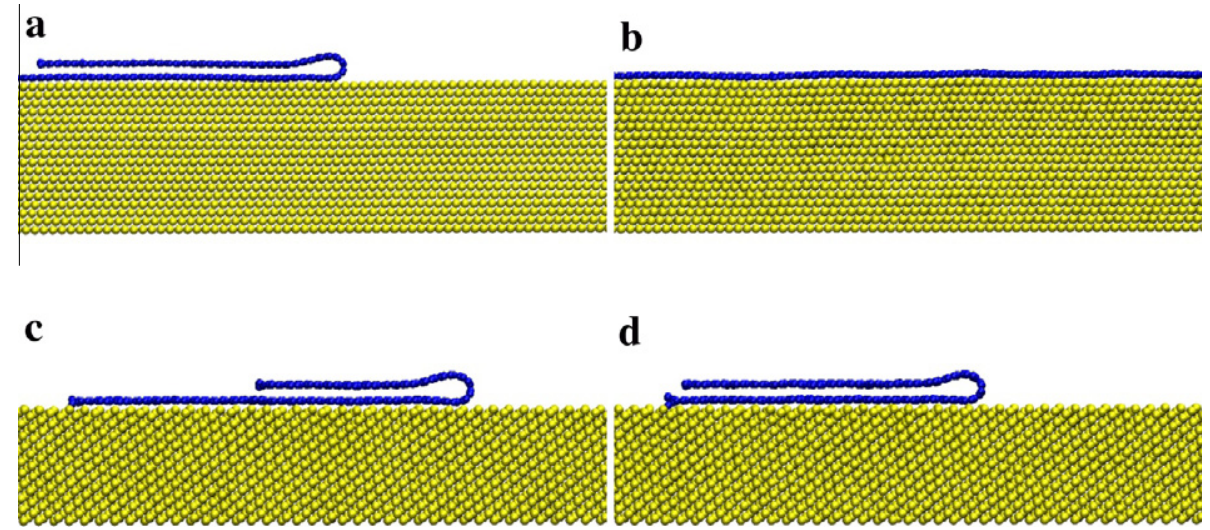

Fig. 6 - (a,b) Initially folded graphene on $\mathrm{Cu}(111)$ finally unfolds. (c,d) Initially partial folded graphene finally folds fully on $\mathrm{Cu}(112)$. The atoms of graphene are in blue color and $\mathrm{Cu}$ in yellow. (For interpretation of the references to color in this figure legend, the reader is referred to the web version of this article.)

between graphene and $\mathrm{Cu}$ surfaces follow the order of $\mathrm{Cu}(111)>(100)>(110)>(112)$. Graphene on Cu surfaces has a preferred orientation: for graphene on $\mathrm{Cu}(111)$, the $0^{\circ}$ orientation is the most energetically stable situation, indicating the growth of graphene on (111) surface of single crystalline $\mathrm{Cu}$ may reduce the formation of grain defects. Furthermore, we show that the conventional peeling model is still applicable to peeling tests of graphene from $\mathrm{Cu}$ substrate. The agreement of $\mathrm{MD}$ results with theoretical predictions indicates that the peeling model can be used to explore interfacial properties between graphene and $\mathrm{Cu}$ surfaces. By identifying the variation of peeling forces with peeling distance, we developed a feasible method to distinguish the layers of graphene being peeled off. The highly oscillating friction force indicates the occurrence of sticking-slipping of graphenes. Through theoretical analysis, we found the position of stiction is controlled by the bending stiffness of graphenes which depends on the number of atomic layers in graphene. Thermal undulation 
also plays a significant role for the dynamical interaction between graphene peeled off and that residing on the substrate. At the end, we investigated the folding/unfolding of graphene on $\mathrm{Cu}$ surfaces. The results show that on $\mathrm{Cu}(111)$ the graphene would not fold yet on $\mathrm{Cu}(112)$ it can. The results indicate that to avoid the folding of graphene edge, the surface with high interfacial binding energy is preferred.

\section{Acknowledgments}

The work is supported by the National Natural Science Foundation of China (NSFC) (Grant Nos. 11023001 and 11021262) and the Chinese Academy of Sciences (CAS) via the Hundred Talent Program, and computation is mainly supported by the Supercomputing Center of Chinese Academy of Sciences (SCCAS) and Shanghai Supercomputer Center (SSC).

\section{R E F E R E N C E S}

[1] Novoselov KS, Geim AK, Morozov SV, Jiang D, Katsnelson MI, Grigorieva IV, et al. Two-dimensional gas of massless dirac fermions in graphene. Nature 2005;438:197-200.

[2] Geim AK, Novoselov KS. The rise of graphene. Nat Mater 2007;6:183-91.

[3] Balandin AA, Ghosh S, Bao W, Calizo I, Teweldebrhan D, Miao F, et al. Superior thermal conductivity of single-layer graphene. Nano Lett 2008;8:902-7.

[4] Bunch JS, van der Zande AM, Verbridge SS, Frank IW, Tanenbaum DM, Parpia JM, et al. Electromechanical resonators from graphene sheets. Science 2007;315: 490-3.

[5] Lee C, Wei X, Kysar JW, Hone J. Measurement of the elastic properties and intrinsic strength of monolayer graphene. Science 2008;321:385-8.

[6] Han MY, Ozyilmaz B, Zhang Y, Kim P. Energy band gap engineering of graphene nanoribbons. Phys Rev Lett 2007;98:206805.

[7] Li X, Wang X, Zhang L, Lee S, Dai H. Chemically derived, ultrasmooth graphene nanoribbon semiconductors. Science 2008;319:1229-32.

[8] Sutter P. Epitaxial graphene: how silicon leaves the scene. Nat Mater 2009;8:171-2.

[9] Kim KS, Zhao Y, Jang H, Lee SY, Kim JM, Kim KS, et al. Largescale pattern growth of graphene films for stretchable transparent electrodes. Nature 2009;457:706-10.

[10] Li X, Cai W, An J, Kim S, Nah J, Yang D, et al. Large-area synthesis of high-quality and uniform graphene films on copper foils. Science 2009;324:1312-4.

[11] Bae S, Kim H, Lee Y, Xu X, Park JS, Zheng Y, et al. Roll-to-roll production of 30-inch graphene films for transparent electrodes. Nature Nanotechnol 2010;5:574-8.

[12] Huang PY, Ruiz-Vargas CS, van der Zande AM, Whitney WS, Levendorf MP, Kevek JW, et al. Grains and grain boundaries in single-layer graphene atomic patchwork quilts. Nature 2011;469:389-92.

[13] Kim K, Lee Z, Regan W, Kisielowski C, Crommie MF, Zettl A. Grain boundary mapping in polycrystalline graphene. ACS Nano 2011;5:2142-6.

[14] Yazyev OV, Louie SG. Topological defects in graphene: dislocations and grain boundaries. Phys Rev B 2010;81:195420.
[15] Grantab R, Shenoy VB, Ruoff RS. Anomalous strength characteristics of tilt grain boundaries in graphene. Science 2010;330:946-8.

[16] Wei Y, Wu J, Yin H, Shi X. Grain misorientation and grainboundary rotation dependent mechanical properties in polycrystalline graphene, submitted for publication.

[17] Lenski DR, Fuhrer MS. Raman and optical characterization of multilayer turbostratic graphene grown via chemical vapor deposition. J Appl Phys 2011;110:013720.

[18] Liu Z, Suenaga K, Harris PJF, Iijima S. Open and closed edges of graphene layers. Phys Rev Lett 2009;102:015501.

[19] Zhang J, Xiao J, Meng X, Monroe C, Huang Y, Zuo JM. Free folding of suspended graphene sheets by random mechanical stimulation. Phys Rev Lett 2010;104: 166805.

[20] Warner JH, Rümmeli MH, Bachmatiuk A, Büchner B. Examining the stability of folded graphene edges against electron beam induced sputtering with atomic resolution. Nanotechnology 2010;21:325702-7.

[21] Kim K, Lee Z, Malone BD, Chan KT, Alemán B, Regan W, et al. Multiply folded graphene. Phys Rev B 2011;83: 245422.

[22] Feng J, Qi L, Huang JY, Li J. Geometric and electronic structure of graphene bilayer edges. Phys Rev B 2009;80:165407.

[23] Rotkin SV, Gogotsi Y. Analysis of non-planar graphitic structures: from arched edge planes of graphite crystals to nanotubes. Mater Res Innovations 2002;5:191-200.

[24] Li X, Zhu Y, Cai W, Borysiak M, Han B, Chen D, et al. Transfer of large-area graphene films for high-performance transparent conductive electrodes. Nano Lett 2009;9: 4359-63.

[25] Regan W, Alem N, Alemán B, Geng B, Girit C, Maserati L, et al. A direct transfer of layer-area graphene. Appl Phys Lett 2010;96:113102.

[26] Brenner DW. The art and science of an analytic potential. Phys Stat Sol 2000;217:23-40.

[27] Pauling L. The nature of the chemical bond. New York: Cornell University Press; 1960.

[28] Foiles SM, Baskes MI, Daw MS. Embedded-atom-method functions for the fcc metals $\mathrm{Cu}, \mathrm{Ag}, \mathrm{Au}, \mathrm{Ni}$. Pd. Pt and their alloys. Phys Rev B 1986;33:7983-91.

[29] Guo Y, Guo W. Structural transformation of partially confined copper nanowires inside defected carbon nanotubes. Nanotechnology 2006;17:4726-30.

[30] Guo Y, Kong Y, Guo W, Gao H. Structural transition of copper nanowires confined in single-walled carbon nanotubes. J Comp Theor Nanosci 2004;1:93-8.

[31] Xu Z, Buehler MJ. Interface structure and mechanics between graphene and metal substrates: a first-principles study. J Phys Condens Matter 2010;22:485301-5.

[32] Gao L, Guest JR, Guisinger NP. Epitaxial graphene on $\mathrm{Cu}(111)$. Nano Lett 2010;10:3512-6.

[33] Spies GJ. The peeling test on redux-bonded joints. J Aircraft Eng 1953;25:64-70.

[34] Wei Y. Thin layer splitting along the elastic-plastic solid surface. Int J Fracture 2002;113:233-52.

[35] Kendall K. Thin-film peeling-the elastic term. J Phys D Appl Phys 1975;8:1449-52.

[36] Mikata Y. Complete solution of elastica for a clamped-hinged beam, and its applications to a carbon nanotube. Acta Mech 2007;190:133-50.

[37] Zhou W, Huang Y, Liu B, Hwang KC, Zuo JM, Buehler MJ, et al. Self-folding of single- and multiwall carbon nanotubes. Appl Phys Lett 2007;90:093107.

[38] Cranford S, Sen D, Buehler MJ. Meso-origami: folding multilayer graphene sheets. Appl Phys Lett 2009;95:123121.

[39] Lu Q, Arroyo M, Huang R. Elastic bending modulus of monolayer graphene. J Phys D Appl Phys 2009;42:102002. 
[40] Lin Y, Inamdar M, Freund LB. The competition between Brownian motion and adhesion in soft materials. J Mech Phys Solids 2008;56:241-50.

[41] Guo Z, Chang TC, Guo XM, Gao HJ. Thermal-induced edge barriers and forces in interlayer interaction of concentric carbon nanotubes. Phys Rev Lett 2011;107:105502.

[42] Zhang DB, Akatyeva E, Dumitrică T. Bending ultrathin graphene at the margins of continuum mechanics. Phys Rev Lett 2011;106:255503.
[43] Li Q, Dong Y, Perez D, Martini A, Carpick RW. Speed dependence of atomic stick-slip friction in optimally matched experiments and molecular dynamics simulations. Phys Rev Lett 2011;106:126101.

[44] Riedo E, Gnecco E, Bennewitz R, Meyer E, Brune H. Interaction potential and attempt frequency governing sliding friction. Phys Rev Lett 2003;91:084502.

[45] Schirmeisen A, Jansen L, Fuchs H. Tip-jump statistics of stick-slip friction. Phys Rev B 2005;71:245403. 